\title{
Peroxisome proliferator-activated receptor- $\alpha$ staining is associated with worse outcome in colorectal liver metastases
}

\author{
TONY PANG ${ }^{1,2}$, ANTONY KAUFMAN ${ }^{3}$, JULIAN CHOI $^{1}$, ANTHONY GILL $^{2-4}$, \\ MARTIN DRUMMOND ${ }^{1}$, THOMAS HUGH ${ }^{1,2}$ and JASWINDER SAMRA ${ }^{1,2,5}$ \\ ${ }^{1}$ Upper Gastrointestinal Surgical Unit, Royal North Shore Hospital and North Shore Private Hospital; \\ ${ }^{2}$ Northern Clinical School, University of Sydney; ${ }^{3}$ Department of Anatomical Pathology, Royal North Shore Hospital; \\ ${ }^{4}$ Cancer Diagnosis and Pathology Group, Kolling Institute of Medicine, Royal North Shore Hospital, St. Leonards, NSW 2065; \\ ${ }^{5}$ Australian School of Advanced Medicine, Macquarie University, Macquarie Park, NSW 2109, Australia
}

Received October 16, 2014; Accepted December 9, 2014

DOI: $10.3892 / \mathrm{mco} .2014 .482$

\begin{abstract}
Peroxisome proliferator-activated receptors (PPARs) are a family of nuclear receptors involved in lipid metabolism and liver response to injury. We hypothesised that differences in the expression of PPARs may reflect differences in the cellular microenvironment of the liver and, consequently, in the behaviour of colorectal liver metastases. Of the 145 patients who underwent hepatectomy for colorectal liver metastases between 1998 and 2007, 103 had adequate tissue for PPAR staining and histological re-evaluation. The histological characteristics evaluated included sinusoidal dilatation, perisinusoidal fibrosis, ballooning and steatosis. PPAR- $\alpha$ and $-\gamma$ staining was performed and the results were correlated with clinical and survival data. Lobular inflammation and sinusoidal dilatation were the most common histopathological abnormalities. A total of $50 \%$ of the patients were PPAR- $\alpha$-negative and $34 \%$ were PPAR- $\gamma$-negative. More patients exhibited lobular inflammation in the PPAR- $\alpha$-positive group $(\mathrm{P}=0.023)$ compared to patients with negative PPAR- $\alpha$ staining, as seen on the multivariate analysis. PPAR- $\gamma$ positivity was associated with oxaliplatin use, surgical margins $\geq 1 \mathrm{~mm}$ and a trend towards a lesser degree of fibrosis. The median follow-up in this cohort of patients was 48 months. Patients with PPAR- $\alpha$ staining had a worse overall survival (median, 36 vs. 79 months, $\mathrm{P}=0.037$ ) compared to those with no PPAR- $\alpha$ staining. There was no correlation between PPAR- $\alpha$ or $-\gamma$ positivity and disease-free survival. In conclusion, PPAR- $\alpha$ staining is associated with lobular inflammation and worse
\end{abstract}

Correspondence to: Professor Jaswinder Samra or Dr Tony Pang, Upper Gastrointestinal Surgical Unit, Royal North Shore Hospital, Reserve Road, St. Leonards, New South Wales 2065, Australia

E-mail: jas.samra@bigpond.com; jass420@hotmail.com

E-mail: tony.pang@sydney.edu.au

Key words: colorectal cancer, peroxisome proliferator-activated receptors, liver metastases, cancer microenvironment overall survival in patients with colorectal liver metastases. The exact mechanism underlying this finding remains unclear and further research into the diagnostic and therapeutic implications is required.

\section{Introduction}

Colorectal cancer is the third most common cancer among men and women in the USA and Australia (1). Approximately $15 \%$ of the patients present with synchronous and a further $15-20 \%$ develop metachronous metastasis (2). The treatment of liver metastasis has radically changed with the advent of novel chemotherapeutic drugs and advances in resection techniques. Despite comprehensive genetic characterization of human colon and rectal cancer (3), the exact mechanisms underlying the metastatic process remain to be elucidated. Recent expansions in the field of metabolomics and the effect on genomics has led to a reinforcement of the idea that the hepatic cellular environment is a crucial factor affecting the behaviour of colorectal liver metastases (4).

Pre-existing liver disease may modify the local environment, thereby altering the ability of circulating tumour cells to form metastatic deposits within the liver. For example, colorectal liver metastases rarely occur in patients with liver cirrhosis $(3,5)$. Systemic chemotherapy may result in liver damage, which may vary from steatosis, to sinusoidal injury, to hepatic fibrosis (6-10). Indeed, a recent study demonstrated that sinusoidal injury was associated with poor survival and early recurrence in patients treated with oxaliplatin-based neoadjuvant therapy (11). The histological changes induced in the liver by chemotherapy have been well documented. However, the local cellular processes resulting in these histopathological characteristics and the altered propensity for hepatic metastasis, are poorly understood.

The liver plays a central role in protein, carbohydrate and lipid metabolism and a number of these metabolic pathways are tightly regulated by receptors. Peroxisome proliferator-activated receptors (PPARs) constitute one such family of nuclear receptors, which regulate lipid metabolism as well as inflammation and response to injury (12). These receptors also play an important role in tumourigenesis (13). Given the 
abovementioned functions, we hypothesised that PPARs may be involved in chemotherapy-induced liver injury and may affect tumour progression and patient survival. Therefore, the aim of this study was to investigate the association of PPAR expression with the histopathological characteristics of chemotherapy-related liver injury and clinical outcomes.

\section{Materials and methods}

Patients. A total of 145 patients underwent hepatectomy for colorectal liver metastases at the North Shore campus of the University of Sydney between June, 1998 and August, 2007. Among those patients, 103 non-consecutive and non-selected patients had adequate tissue blocks for PPAR staining and histological re-evaluation; this cohort formed the basis of the present study. Basic demographic, clinical and pathological data were collected from hospital records, while survival data were collected from ongoing clinical follow-up. Patients were excluded from the study if they had undergone prior liver resection, whereas those who underwent subsequent resection were included. Synchronous liver metastases were defined as those presenting within 4 months of the primary colorectal cancer diagnosis; metachronous liver metastases were defined as those identified $>4$ months after the primary colorectal cancer diagnosis.

Liver resection. All the patients underwent a standard preoperative assessment, which included a multiphase computed tomography (CT) scan of the abdomen and thorax. A positron emission tomography scan was also performed from January, 2004 onwards. All the cases were discussed at a multidisciplinary group meeting prior to liver resection. The operative criteria included the likelihood of achieving an $\mathrm{R} 0$ resection, along with preservation of vascular inflow and outflow and an adequate post-resection residual liver volume. Patients with limited extrahepatic intra-abdominal disease were not excluded from resection. Liver transection was performed using the Cavitron Ultrasonic Surgical Aspirator dissection device (Integra, Plainsboro, NJ, USA), under low central venous pressure conditions, with intermittent inflow occlusion. The postoperative complications were classified according to the Clavien-Dindo classification, with major complications graded as $\geq 3$ (14). The follow-up regime included 3-monthly clinical evaluations, serum tumour markers and CT scans of the abdomen and thorax for the first year, 6-monthly in the second year and annually thereafter.

Pathological protocol. The histological analyses were based on hematoxylin and eosin, Masson trichrome and reticulin stains. The following characteristics were evaluated and scored: sinusoidal dilatation, perisinusoidal fibrosis, ballooning and steatosis and were graded semi-quantitatively as follows: 0, absent; 1, mild (centrilobular involvement limited to one-third of the lobule); 2, moderate (centrilobular involvement involving two-thirds of the lobule); and 3, severe (complete lobular involvement). Steatosis was estimated as the percentage of involved hepatocytes and was categorized as follows: 0, absent; 1 , mild (steatosis in $<30 \%$ of the hepatocytes); 2 , moderate (steatosis in 30-60\% of the hepatocytes); and 3 , severe (steatosis in $>60 \%$ of the hepatocytes). These variables were scored by a single pathologist (A.K.) who was blinded as to all other data.

For PPAR $-\gamma$ and $-\alpha$ staining, the slides were processed with an automated staining system, namely the Bond-Max Autostainer (Vision Biosystems, Mount Waverley, Victoria, Australia) used according to the manufacturer's protocol and with the manufacturer's retrieval solutions. For PPAR- $\alpha$, a rabbit polyclonal antibody (cat. no. 8934; Abcam, Cambridge, UK) was used at a dilution of 1:600. For PPAR- $\gamma$, a mouse monoclonal antibody (clone E8; cat. no. SC-7273; Santa Cruz Biotechnology, Santa Cruz, CA, USA) was used at a dilution of 1:100. For both antibodies, heat-induced epitope retrieval was performed for $30 \mathrm{~min}$ in the manufacturer's alkaline retrieval solution ER2 (VBS part no. AR9640). A biotin-free detection system was employed (VBS part no. DS 9713).

The PPAR- $\gamma$ and $-\alpha$ slides were scored semi-quantitatively as follows: 0 , no positive cells $(<1 \%) ; 1+$, focal positive staining $(<10 \%$ of cells); $2+$, diffuse weak staining $(>10 \%$ of cells staining weakly positive); and $3+$, diffuse strong positive staining ( $>10 \%$ of cells staining diffusely strongly positive). PPAR $-\gamma$ and $-\alpha$ staining were scored by a single pathologist (A.G.) who was blinded as to all other data.

Statistical analysis. Descriptive statistics were reported using means (standard deviation) and medians (interquartile ranges), depending on the distribution. Inferential statistical comparisons between groups were performed using the Fisher's exact test and Student's t-test for categorical and parametric data, respectively. A multivariate logistic analysis of covariates with $\mathrm{P}<0.2$ in the univariate analysis was performed. Hosmer and Lemeshow's goodness-of-fit test was used.

Overall survival was defined as survival censored by either last follow-up or death from any cause. Disease-free survival was censored at the first event of recurrence in any area, last follow-up or death. Kaplan-Meier curves were constructed. A univariate survival analysis was performed using the log-rank test and a multivariate analysis was performed by constructing Cox proportional hazards models from covariates with $\mathrm{P}<0.2$ in the univariate analysis. The purposeful selection of covariates method was used to select variables for the final model. The final model was then assessed for validity of the proportional hazards assumption using Shoenfeld residuals and goodness-of-fit using Cox-Snell residuals. All the statistical analyses were performed using Stata/SE software, version 11.2 for Windows (StataCorp, College Station, TX, USA).

In order to investigate factors affecting long-term survival, a survival analysis was performed excluding early postoperative deaths (90-day mortality); i.e., conditional upon survival to 90 days.

\section{Results}

Clinicopathological characteristics. The demographic, histopathological and clinical characteristics of the study cohort are summarized in Table I. Briefly, the mean age of the patients was 63 years and there were $68(66 \%)$ men and $35(34 \%)$ women. Lobular inflammation and sinusoidal dilatation were the most common histopathological abnormalities, with $>70 \%$ of the patients exhibiting some degree of lobular inflammation 
Table I. Summary of patient characteristics for the entire cohort and according to PPAR- $\alpha$ and- $\gamma$ staining.

\begin{tabular}{|c|c|c|c|c|c|c|c|}
\hline \multirow[b]{2}{*}{ Covariates } & \multirow[b]{2}{*}{ Overall } & \multicolumn{3}{|c|}{ PPAR- $\alpha^{\mathrm{a}}$} & \multicolumn{3}{|c|}{ PPAR- $\gamma^{\mathrm{b}}$} \\
\hline & & Negative & Positive $^{c}$ & P-value & Negative & Positive $^{b}$ & P-value \\
\hline \multicolumn{8}{|l|}{ Patient characteristics } \\
\hline \multirow{2}{*}{\multicolumn{2}{|c|}{$\begin{array}{l}\text { Age, years [mean }(95 \% \text { CI })] 63(11) \\
\text { Gender }(n, \%)\end{array}$}} & $63(60-66)$ & $63(59-66)$ & 0.96 & $61(58-65)$ & $64(61-66)$ & 0.31 \\
\hline & & & & & & & 0.046 \\
\hline Female & $35(34)$ & $19(37)$ & $16(31)$ & 0.68 & $7(21)$ & $28(42)$ & \\
\hline Male & $68(66)$ & $32(63)$ & 35 (69) & & $27(79)$ & $39(58)$ & \\
\hline \multicolumn{8}{|c|}{ Pathological characteristics $(\mathrm{n}, \%)$} \\
\hline Steatosis & & & & 0.92 & & & 0.59 \\
\hline 0 & $54(52)$ & $28(55)$ & $25(49)$ & & $15(44)$ & $37(56)$ & \\
\hline 1 & $25(24)$ & $12(24)$ & $13(25)$ & & $11(32)$ & $14(21)$ & \\
\hline 2 & $21(20)$ & $10(20)$ & $11(22)$ & & $7(21)$ & $14(21)$ & \\
\hline 3 & $3(3)$ & $1(2)$ & $2(4)$ & & $1(3)$ & $2(3)$ & \\
\hline \multicolumn{2}{|l|}{ Lobular inflammation } & & & $\mathbf{0 . 0 2 3}^{\mathrm{b}}$ & & & 0.15 \\
\hline 0 & $30(29)$ & $20(39)$ & $10(20)$ & & $6(18)$ & $24(36)$ & \\
\hline 1 & $38(37)$ & $19(37)$ & $18(35)$ & & $16(47)$ & $20(30)$ & \\
\hline 2 & $24(23)$ & $6(12)$ & $18(35)$ & & $7(21)$ & $17(25)$ & \\
\hline 3 & $11(11)$ & $6(12)$ & $5(10)$ & & $5(15)$ & $6(9)$ & \\
\hline \multicolumn{2}{|l|}{ Ballooning } & & & 1.00 & & & 0.93 \\
\hline 0 & $81(79)$ & $40(78)$ & $40(78)$ & & $27(79)$ & $52(78)$ & \\
\hline 1 & 17 (17) & $9(18)$ & $8(16)$ & & $6(18)$ & $11(16)$ & \\
\hline 2 & $5(5)$ & $2(4)$ & $3(6)$ & & $1(3)$ & $4(6)$ & \\
\hline \multicolumn{2}{|l|}{ Fibrosis } & & & 0.36 & & & 0.23 \\
\hline 0 & $52(51)$ & $27(53)$ & $24(47)$ & & $15(44)$ & $35(52)$ & \\
\hline 1 & $22(21)$ & $13(25)$ & $9(18)$ & & $5(15)$ & $17(25)$ & $0 / 1$ vs. $2 / 3$ \\
\hline 2 & $21(20)$ & 7 (14) & $14(27)$ & & $10(29)$ & $11(16)$ & $P=0.042$ \\
\hline 3 & $8(8)$ & $4(8)$ & $4(8)$ & & $4(12)$ & $4(6)$ & \\
\hline \multicolumn{2}{|l|}{ Sinusoidal dilatation } & & & 0.65 & & & 0.24 \\
\hline 0 & $29(28)$ & $17(33)$ & $12(24)$ & & $6(18)$ & $22(33)$ & \\
\hline 1 & $32(31)$ & $14(27)$ & $17(33)$ & & $10(29)$ & $21(31)$ & \\
\hline 2 & 17 (17) & $7(14)$ & $10(20)$ & & $6(18)$ & $11(16)$ & \\
\hline 3 & $25(24)$ & $13(26)$ & $12(24)$ & & $12(35)$ & $13(19)$ & \\
\hline \multicolumn{8}{|l|}{ PPAR staining $(\mathrm{n}, \%)$} \\
\hline \multicolumn{8}{|l|}{ PPAR- $\alpha$} \\
\hline 0 & $51(50)$ & & & & & & \\
\hline $1+$ & $24(24)$ & & & & & & \\
\hline $2+$ & $11(11)$ & & & & & & \\
\hline $3+$ & $16(16)$ & & & & & & \\
\hline \multicolumn{8}{|l|}{ PPAR- $\gamma$} \\
\hline 0 & $34(34)$ & & & & & & \\
\hline $1+$ & $52(51)$ & & & & & & \\
\hline $2+$ & $14(14)$ & & & & & & \\
\hline $3+$ & $1(1)$ & & & & & & \\
\hline \multicolumn{8}{|c|}{ Clinical characteristics $(\mathrm{n}, \%)$} \\
\hline \multicolumn{2}{|l|}{ Resection type } & & & 1.00 & & & 0.40 \\
\hline Minor & $45(44)$ & $22(43)$ & $22(43)$ & & $12(35)$ & $31(46)$ & \\
\hline Major & $58(56)$ & $29(57)$ & $29(57)$ & & $22(65)$ & $36(54)$ & \\
\hline \multicolumn{2}{|l|}{ Resection margin (mm) } & & & 0.93 & & & 0.015 \\
\hline $\mathrm{R} 1$ & 17 (16) & $9(18)$ & $8(16)$ & & $9(26)$ & $8(12)$ & \\
\hline \multicolumn{8}{|l|}{ R0 } \\
\hline$<1$ & 14 (14) & $8(16)$ & $6(12)$ & & $8(24)$ & $6(9)$ & \\
\hline $1-10$ & $48(47)$ & $23(45)$ & $24(47)$ & & $9(26)$ & $37(55)$ & \\
\hline$>10$ & $24(23)$ & $11(22)$ & $13(25)$ & & $8(24)$ & $16(24)$ & \\
\hline
\end{tabular}


Table I. Continued.

\begin{tabular}{|c|c|c|c|c|c|c|c|}
\hline \multirow[b]{2}{*}{ Covariate } & \multirow[b]{2}{*}{ Overall } & \multicolumn{3}{|c|}{ PPAR- $\alpha^{\mathrm{a}}$} & \multicolumn{3}{|c|}{ PPAR- $\gamma^{\mathrm{b}}$} \\
\hline & & Negative & Positive $^{c}$ & P-value & Negative & Positive $^{c}$ & P-value \\
\hline Preoperative chemotherapy & & & & 1.00 & & & 0.39 \\
\hline No & $40(39)$ & $20(39)$ & $20(40)$ & & $15(45)$ & $24(36)$ & \\
\hline Yes & $62(61)$ & $31(61)$ & $30(60)$ & & $18(55)$ & $43(64)$ & \\
\hline Preoperative oxaliplatin & & & & 0.64 & & & 0.023 \\
\hline No & $80(78)$ & $38(75)$ & $41(80)$ & & $31(91)$ & $47(70)$ & \\
\hline Yes & $23(22)$ & $13(25)$ & $10(20)$ & & $3(9)$ & $20(30)$ & \\
\hline Postoperative chemotherapy & & & & 1.00 & & & 1.00 \\
\hline No & $50(50)$ & $24(49)$ & $26(51)$ & & $17(50)$ & $33(51)$ & \\
\hline Yes & $51(51)$ & $25(51)$ & $25(49)$ & & $17(50)$ & $32(49)$ & \\
\hline Complications (Clavien-Dindo) & & & & 0.086 & & & 0.54 \\
\hline$<3$ & $87(84)$ & $46(90)$ & $40(78)$ & & $29(85)$ & $56(84)$ & \\
\hline$\geq 3$ & $16(16)$ & $5(10)$ & $11(22)$ & & $5(15)$ & $11(16)$ & \\
\hline Perioperative death & & & & 0.31 & & & 0.59 \\
\hline No & $98(96)$ & $50(98)$ & $48(94)$ & & $33(97)$ & $64(96)$ & \\
\hline Yes & $4(4)$ & $1(2)$ & $3(6)$ & & $1(3)$ & $3(4)$ & \\
\hline \multicolumn{8}{|c|}{ Primary tumour characteristics $(\mathrm{n}, \%)$} \\
\hline Metachronous & & & & 0.53 & & & 0.53 \\
\hline No & $49(48)$ & $23(45)$ & $26(52)$ & & $14(42)$ & $34(51)$ & \\
\hline Yes & $53(52)$ & $28(55)$ & $24(48)$ & & $19(58)$ & $33(49)$ & \\
\hline Lymph node + & & & & 0.55 & & & 0.53 \\
\hline No & $44(44)$ & $20(40)$ & $24(48)$ & & $13(39)$ & $31(47)$ & \\
\hline Yes & $57(56)$ & $30(60)$ & $26(52)$ & & $20(61)$ & $35(53)$ & \\
\hline \multicolumn{8}{|l|}{ Tumour characteristics } \\
\hline No. of metastases (n, \%) & & & & 0.077 & & & 0.92 \\
\hline 1 & $54(52)$ & $29(57)$ & $24(47)$ & & $19(56)$ & $34(51)$ & \\
\hline $2-3$ & $34(33)$ & $12(23)$ & $22(43)$ & & $10(29)$ & $23(34)$ & \\
\hline$>3$ & $15(15)$ & $10(20)$ & $5(10)$ & & $5(15)$ & $10(15)$ & \\
\hline Grade of differentiation (n, \%) & & & & 0.24 & & & 0.25 \\
\hline High & $10(11)$ & $3(6)$ & $7(15)$ & & $5(17)$ & $4(6)$ & \\
\hline Moderate & $76(81)$ & $39(83)$ & $36(80)$ & & $24(80)$ & $52(84)$ & \\
\hline Poor & $8(8.5)$ & $5(11)$ & $2(4)$ & & $1(3)$ & $6(10)$ & \\
\hline Size, mm [mean (range)] & $46(28)$ & $46(37-55)$ & $45(38-52)$ & 0.86 & $49(39-59)$ & $44(37-50)$ & 0.40 \\
\hline
\end{tabular}

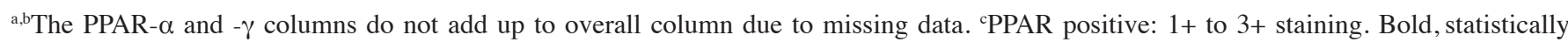
significant. PPAR, peroxisome proliferator-activated receptor.

and approximately the same proportion exhibiting some degree of sinusoidal dilatation. The least common abnormality was ballooning (only $21 \%$ of the patients). In terms of tumour characteristics, the majority (52\%) were solitary metastases and most of the tumours $(76 \%)$ were moderately differentiated. The mean tumour size was $46 \mathrm{~mm}$ and $84 \%$ of the patients underwent an $\mathrm{R} 0$ resection.

The PPAR- $\alpha$ and $-\gamma$ staining characteristics are shown in Table I. A total of $50 \%$ of the patients in this study were PPAR- $\alpha$-negative and $34 \%$ were PPAR- $\gamma$-negative. When comparing patients whose livers stained for PPAR- $\alpha$ to those who did not (Table I), there was a significant difference in the proportion of patients with lobular inflammation, with more patients exhibiting lobular inflammation in the PPAR- $\alpha$-positive group $(\mathrm{P}=0.023)$. As regards PPAR $-\gamma$ staining, there were more patients with moderate to severe fibrosis (score 2 or 3 ) in the PPAR- $\gamma$-negative group compared to the PPAR- $\gamma$-positive group. On multivariate analysis (Table II), PPAR- $\alpha$ staining was significantly associated with lobular inflammation (odds ratio $=2.9$ ). There was a non-significant trend with regards to fibrosis, whilst the association with oxaliplatin use exhibited marginal statistical significance.

The median follow-up period for the study cohort was 48 months (range, 0.4-142 months). The results of the univariate survival analysis demonstrated improved survival associated with PPAR- $\alpha$ negativity (median survival, 79 vs. 36 months; $\mathrm{P}=0.037$ ), minor resection and the absence of major complications (Table III). 

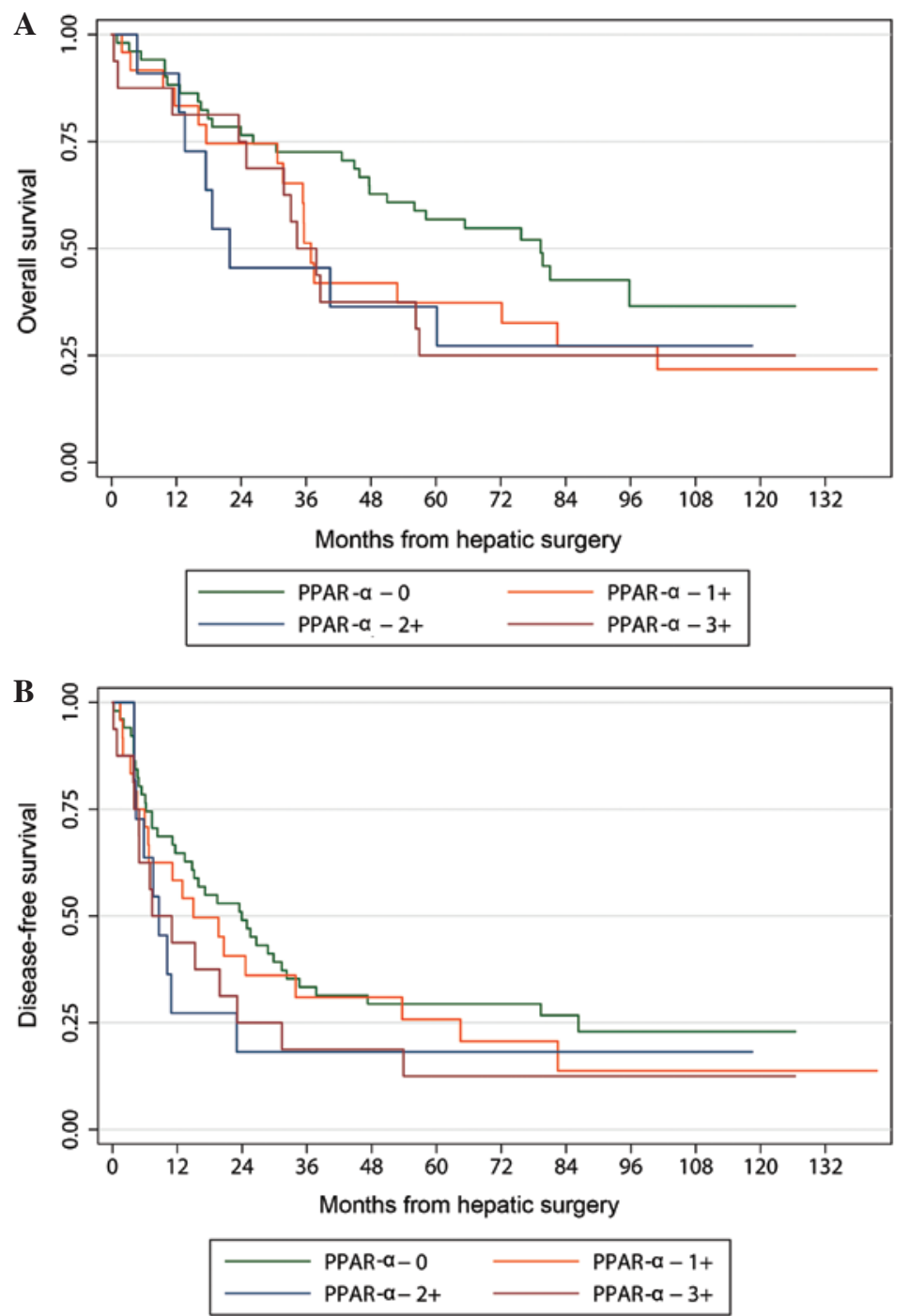

Figure 1. Kaplan-Meier curves illustrating the effect of PPAR- $\alpha$ staining on (A) overall survival and (B) disease-free survival. PPAR, peroxisome proliferator-activated receptor.

Table II. Multivariate logistic regression model for the expression of PPAR- $\alpha$ and $-\gamma$.

\begin{tabular}{lllcl}
\hline Covariates & OR & SE & $95 \%$ CI & P-value \\
\hline $\begin{array}{l}\text { PPAR- } \alpha \\
\quad \text { Lobular inflammation }\end{array}$ & 2.9 & 1.3 & $1.2-7.0$ & $\mathbf{0 . 0 1 5}$ \\
$\quad$ Clavien-Dindo $\geq 3$ & 3.0 & 1.8 & $0.92-9.7$ & 0.067 \\
PPAR- $\gamma$ & & & & \\
$\quad$ Oxaliplatin use & 3.9 & 2.7 & $1.0-15$ & $\mathbf{0 . 0 4 8}$ \\
Margin $(\geq 1$ vs. $<1 \mathrm{~mm})$ & 3.3 & 1.6 & $1.3-8.6$ & $\mathbf{0 . 0 1 5}$ \\
Fibrosis (score 3/4 vs. 0/1) & 0.40 & 0.20 & $0.15-1.1$ & 0.070 \\
Male gender & 0.41 & 0.22 & $0.15-1.2$ & 0.091 \\
\end{tabular}

Bold,statistically significant.PPAR, peroxisome proliferator-activated receptor; OR, odds ratio; $\mathrm{SE}$, standard error; $\mathrm{CI}$, confidence interval.

The median disease-free survival was 15 months. The factors associated with prolonged disease-free survival on univariate analysis included having a solitary tumour, tumour size $<40 \mathrm{~mm}$, no postoperative chemotherapy and absence of major postoperative complications. Neither PPAR- $\alpha$ nor PPAR- $\gamma$ positivity were associated with disease-free survival. Following exclusion of early postoperative deaths, the P-value associated with major complications increased to 0.042 and 0.17 for overall and disease-free survival, respectively, suggesting that early deaths may have accounted for the apparent association.

The multivariate analysis confirmed an independent association between PPAR- $\alpha$ staining and overall survival. However, this was only of marginal statistical significance (Table IV). The Kaplan-Meier curve comparing different levels of PPAR- $\alpha$ staining demonstrated that survival was similar in each of the PPAR- $\alpha$ groups. However, when PPAR- $\alpha$ expression was not detected, survival was significantly better (Fig. 1). PPAR $-\gamma$ staining exhibited no significant association with survival. A multivariate analysis of the determinants of disease-free survival confirmed the conventional poor prognostic factors, such as multiple metastases and large tumour size. In addition, patients who had received postoperative chemotherapy exhibited worse disease-free survival compared to those who did not. 
Table III. Influence of various patient factors and PPAR status on survival.

\begin{tabular}{|c|c|c|c|c|c|}
\hline \multirow[b]{2}{*}{ Covariates } & \multirow[b]{2}{*}{ No. } & \multicolumn{2}{|c|}{ Overall survival } & \multicolumn{2}{|c|}{ Disease-free survival } \\
\hline & & $\begin{array}{l}\text { Median survival, } \\
\text { months }(95 \% \mathrm{CI})\end{array}$ & P-value & $\begin{array}{l}\text { Median survival, } \\
\text { months }(95 \% \mathrm{CI})\end{array}$ & P-value \\
\hline Overall & 103 & $48(36-42)$ & & $15(11-24)$ & \\
\hline \multicolumn{6}{|l|}{ Demographic characteristics } \\
\hline $\begin{array}{l}\text { Age (years) } \\
<65 \\
\geq 65\end{array}$ & $\begin{array}{l}55 \\
48\end{array}$ & $\begin{array}{l}65(38-82) \\
39(32-57)\end{array}$ & 0.44 & $\begin{array}{l}20(7-31) \\
13(8-24)\end{array}$ & 0.82 \\
\hline $\begin{array}{l}\text { Gender } \\
\text { Female } \\
\text { Male }\end{array}$ & $\begin{array}{l}35 \\
68\end{array}$ & $\begin{array}{l}81(32-N R) \\
46(35-60)\end{array}$ & 0.26 & $\begin{array}{l}12(7-82) \\
15(11-23)\end{array}$ & 0.19 \\
\hline \multicolumn{6}{|l|}{ PPAR staining } \\
\hline $\begin{array}{l}\text { PPAR- } \alpha \\
0 \\
1+\text { to } 3+\end{array}$ & $\begin{array}{l}51 \\
51\end{array}$ & $\begin{array}{l}79(48-N R) \\
36(31-56)\end{array}$ & 0.037 & $\begin{array}{l}24(12-32) \\
11(7-20)\end{array}$ & 0.12 \\
\hline $\begin{array}{l}\text { PPAR- } \gamma \\
0 \\
1+\text { to } 3+\end{array}$ & $\begin{array}{l}34 \\
67\end{array}$ & $\begin{array}{l}43(33-60) \\
56(32-82)\end{array}$ & 0.44 & $\begin{array}{l}15(7-25) \\
19(9-27)\end{array}$ & 0.39 \\
\hline \multicolumn{6}{|l|}{ Pathological characteristics } \\
\hline $\begin{array}{l}\text { Steatosis } \\
0-1 \\
2-3\end{array}$ & $\begin{array}{l}79 \\
24\end{array}$ & $\begin{array}{l}53(37-80) \\
44(18-83)\end{array}$ & 0.29 & $\begin{array}{l}14(8-23) \\
25(7-32)\end{array}$ & 0.72 \\
\hline $\begin{array}{l}\text { Lobular inflammation } \\
0-1 \\
2-3\end{array}$ & $\begin{array}{l}68 \\
35\end{array}$ & $\begin{array}{l}53(37-81) \\
40(31-82)\end{array}$ & 0.60 & $\begin{array}{l}15(9-24) \\
20(8-26)\end{array}$ & 0.42 \\
\hline $\begin{array}{l}\text { Ballooning } \\
0 \\
1-2\end{array}$ & $\begin{array}{l}81 \\
22\end{array}$ & $\begin{array}{l}51(36-79) \\
72(19-101)\end{array}$ & 0.92 & $\begin{array}{l}15(8-23) \\
17(8-34)\end{array}$ & 0.73 \\
\hline $\begin{array}{l}\text { Fibrosis } \\
0-1 \\
2-3\end{array}$ & $\begin{array}{l}74 \\
29\end{array}$ & $\begin{array}{l}48(35-72) \\
57(33-82)\end{array}$ & 0.87 & $\begin{array}{l}15(7-24) \\
21(10-31)\end{array}$ & 0.90 \\
\hline $\begin{array}{l}\text { Sinusoidal dilatation } \\
0-1 \\
2-3\end{array}$ & $\begin{array}{l}61 \\
42\end{array}$ & $\begin{array}{l}46(34-82) \\
53(34-81)\end{array}$ & 0.81 & $\begin{array}{l}15(11-24) \\
15(7-31)\end{array}$ & 0.88 \\
\hline \multicolumn{6}{|l|}{ Clinical characteristics } \\
\hline $\begin{array}{l}\text { Resection type } \\
\text { Minor } \\
\text { Major }\end{array}$ & $\begin{array}{l}45 \\
58\end{array}$ & $\begin{array}{l}65(38-N R) \\
39(26-72)\end{array}$ & 0.048 & $\begin{array}{l}20(8-35) \\
15(8-24)\end{array}$ & 0.060 \\
\hline $\begin{array}{l}\text { Resection margin }(\mathrm{mm}) \\
<1 \\
\geq 1\end{array}$ & $\begin{array}{l}31 \\
72\end{array}$ & $\begin{array}{l}36(24-79) \\
57(38-82)\end{array}$ & 0.19 & $\begin{array}{c}8(5-24) \\
20(12-30)\end{array}$ & 0.090 \\
\hline $\begin{array}{l}\text { Preoperative chemotherapy } \\
\text { No } \\
\text { Yes }\end{array}$ & $\begin{array}{l}40 \\
62\end{array}$ & $\begin{array}{l}60(37-101) \\
48(31-72)\end{array}$ & 0.52 & $\begin{array}{l}20(11-38) \\
13(7-23)\end{array}$ & 0.21 \\
\hline $\begin{array}{l}\text { Postoperative chemotherapy } \\
\text { No } \\
\text { Yes }\end{array}$ & $\begin{array}{l}50 \\
51\end{array}$ & $\begin{array}{l}57 \text { (34-NR) } \\
46(34-80)\end{array}$ & 0.56 & $\begin{array}{l}23(10-54) \\
14(7-21)\end{array}$ & $0.043^{\mathrm{a}}$ \\
\hline $\begin{array}{l}\text { Complications } \\
\text { Dindo } \leq 2 \\
\text { Dindo } \geq 3\end{array}$ & $\begin{array}{l}87 \\
16\end{array}$ & $\begin{array}{l}56(40-96) \\
17(2-72)\end{array}$ & 0.0008 & $\begin{array}{c}20(12-25) \\
5(1-13)\end{array}$ & $0.0098^{\mathrm{a}}$ \\
\hline $\begin{array}{l}\text { Complications (excl. deaths) } \\
\text { Dindo } \leq 2 \\
\text { Dindo } \geq 3\end{array}$ & $\begin{array}{l}87 \\
12\end{array}$ & $\begin{array}{l}56(40-96) \\
24(5-81)\end{array}$ & 0.042 & $\begin{array}{c}20(12-25) \\
7(2-54)\end{array}$ & 0.17 \\
\hline
\end{tabular}


Table III. Continued.

\begin{tabular}{|c|c|c|c|c|c|}
\hline \multirow[b]{2}{*}{ Covariates } & \multirow[b]{2}{*}{ No. } & \multicolumn{2}{|c|}{ Overall survival } & \multicolumn{2}{|c|}{ Disease-free survival } \\
\hline & & $\begin{array}{l}\text { Median survival, } \\
\text { months (95\% CI) }\end{array}$ & P-value & $\begin{array}{l}\text { Median survival, } \\
\text { months ( } 95 \% \mathrm{CI} \text { ) }\end{array}$ & P-value \\
\hline \multicolumn{6}{|c|}{ Primary tumour characteristics } \\
\hline Metachronous & & & 0.77 & & 0.46 \\
\hline No & 49 & $51(35-81)$ & & $15(7-25)$ & \\
\hline Yes & 53 & $56(26-101)$ & & $20(10-30)$ & \\
\hline Lymph node + & & & 0.50 & & 0.19 \\
\hline No & 44 & $53(37-101)$ & & $20(10-35)$ & \\
\hline Yes & 57 & $48(31-81)$ & & $14(6-23)$ & \\
\hline \multicolumn{6}{|l|}{ Tumour characteristics } \\
\hline No. of metastases & & & 0.16 & & 0.029 \\
\hline Solitary & 54 & 57 (40-NR) & & $23(12-35)$ & \\
\hline Multiple & 49 & $39(26-72)$ & & $11(6-17)$ & \\
\hline Grade of differentiation & & & 0.41 & & 0.41 \\
\hline High-moderate & 86 & $53(36-79)$ & & $15(8-25)$ & \\
\hline Poor & 8 & $34(2-N R)$ & & $14(1-25)$ & \\
\hline Size (mm) & & & 0.070 & & 0.033 \\
\hline$<40$ & 49 & $65(45-101)$ & & $25(13-35)$ & \\
\hline$\geq 40$ & 54 & $34(25-53)$ & & $11(7-20)$ & \\
\hline
\end{tabular}

${ }^{\mathrm{a}, \mathrm{b}}$ The PPAR- $\alpha$ and $-\gamma$ columns do not add up to overall column due to missing data. Bold, statistically significant. PPAR, peroxisome proliferator-activated receptor; CI, confidence interval; NR, median survival not reached.

Table IV. Multivariate models for long-term survival overall survival and disease-free survival. ${ }^{\mathrm{a}}$

\begin{tabular}{lcc}
\hline Covariates & HR $(95 \% \mathrm{CI})$ & P-value \\
\hline $\begin{array}{l}\text { Overall survival } \\
\text { PPAR- } \alpha+\end{array}$ & $1.68(1.03-2.8)$ & $\mathbf{0 . 0 3 9}$ \\
Margin $\geq 1$ mm & $0.6(0.4-1.0)$ & 0.06 \\
Disease-free survival & & \\
Multiple metastases & $1.7(1.1-2.7)$ & $\mathbf{0 . 0 2 7}$ \\
Size $\geq 40$ mm & $1.7(1.04-2.7)$ & $\mathbf{0 . 0 3 4}$ \\
Postop chemotherapy & $1.7(1.1-2.8)$ & $\mathbf{0 . 0 2 2}$ \\
\hline
\end{tabular}

${ }^{\text {aEarly }}$ postoperative deaths were excluded to avoid factors associated with early postoperative deaths affecting the long-term survival results (i.e., analysis conditional upon survival to 90 days; see 'Materials and methods' for details). Bold, statistically significant. PPAR, peroxisome proliferator-activated receptor; HR, hazard ratio; CI, confidence interval; postop, postoperative.

\section{Discussion}

Two major interrelated issues are raised by this study. First, the effect of chemotherapy on non-neoplastic liver parenchyma and the role of PPARs play in modulating this effect; and second, the role of PPAR- $\alpha$ and $-\gamma$ on tumour progression and, therefore, long-term patient outcomes.
It is well known that preoperative chemotherapy damages the liver parenchyma surrounding the tumour. Microscopically, this damage variously causes steatosis, steatohepatitis, fibrosis and sinusoidal injury (9). In addition, different types of chemotherapy cause different patterns of injury. Specifically, oxaliplatin causes sinusoidal obstruction, whereas irinotecan causes steatohepatitis $(8,15,16)$. However, the effect of chemotherapy-related liver injury on perioperative morbidity and mortality remains unclear, with certain studies reporting an association $(15,17)$, unlike others $(8,16)$. The effects on long-term mortality have been even less extensively investigated. A recent retrospective study of 196 patients who underwent resection of liver metastases reported that severe sinusoidal dilatation, related to oxaliplatin use, was associated with poor overall and recurrence-free survival (11). This has not been previously described. Contrary to that study, we did not observe any association between the histopathological characteristics of chemotherapy-associated liver injury (CALI) and overall survival. Furthermore, we did not identify an association between CALI and perioperative morbidity or mortality (data not shown).

PPAR- $\alpha$ and $-\gamma$ are nuclear receptors. Following ligand binding, heterodimerisation occurs with retinoid $X$ receptor to form a transcription factor with resultant downstream effects (18). PPAR- $\alpha$ is expressed in a wide variety of tissue cells involved in lipid metabolism, including hepatocytes. By contrast, PPAR- $\gamma$ is mainly expressed in adipose tissue and is generally poorly expressed in the liver, although its expression increases with lipid accumulation (19-21). PPAR- $\alpha$ is associated 
with lipid metabolism and inflammation. In addition, the two receptors play a role in the modulation of chemical and ischaemic-reperfusion mediated hepatic damage (18).

The role of these receptors in chemotherapy-associated damage has not been established; however, our hypothesis is that they are likely to be significant. However we did not, in general, observe an association between different types of liver injury and the expression of PPAR- $\alpha$ and $-\gamma$ as detected by immunohistochemistry, with the exception of the association between PPAR- $\alpha$ expression and the presence of lobular inflammation. Of note, PPAR- $\alpha$ generally exerts an anti-inflammatory effect, e.g., in the amelioration of ethanol- and diet-induced steatohepatitis, as well as ischaemic-reperfusion injury in rats (22-25). We therefore hypothesised that PPAR- $\alpha$ expression may be a response to lobular inflammation rather than a causative factor.

In the liver, decreased expression of PPAR- $\gamma$ in hepatic stellate cells is associated with activation and transdifferentiation; this, in turn, leads to hepatic fibrosis (12). However, the role of PPAR- $\gamma$ in hepatocytes is less clear. Indeed, we found that PPAR- $\gamma$ expression was associated with a non-significant trend $(\mathrm{P}=0.07)$ for milder (or absent) hepatic fibrosis. We also noted an unexpected association with good surgical margins, perhaps as a result of differences in liver texture.

Furthermore, we observed that the expression of PPAR- $\alpha$ was associated with worse long-term overall survival compared to tumours that do not express this receptor. This was unexpected, as PPAR- $\alpha$ activity is considered to exert a negative effect on tumourigenesis. This inhibitory effect is likely mediated by at least three different pathways: inhibition of endothelial cell proliferation, anti-inflammatory action and inhibition of the Warburg effect $(13,26,27)$. However, the effect of PPAR- $\alpha$ on tumour growth is likely to be more complex, as PPAR- $\alpha$-deficient hosts (PPAR- $\alpha$ knockout rats) may prevent tumour growth by inhibition of angiogenesis through excessive inflammation (28). Although such an effect has never been demonstrated in human hepatocytes, it confirms a complex interaction between the tumour microenvironment and tumour growth and progression. Larger studies involving molecular analysis are required to confirm the role of these receptors in colorectal liver metastases.

The major weakness of this study is the lack of mechanistic explanation for these associations. Further studies with more mechanistic assays or metabolomic studies are required to investigate this issue.

In summary, we found that PPAR- $\alpha$ is associated with the presence of lobular inflammation. Apart from this finding, neither PPAR- $\alpha$ nor PPAR- $\gamma$ were found to be associated with any specific patterns of chemotherapy-related liver damage. There was also an association between PPAR- $\alpha$ expression and worse overall survival. However, there is no obvious mechanistic explanation for these findings. This study has raised several issues regarding the role PPAR- $\alpha$ plays in tumour progression. The exact underlying mechanisms remain unclear; specifically, it has not been elucidated whether inflammation causes tumour progression and increased PPAR- $\alpha$ expression in the liver, or the increased PPAR expression results in increased inflammatory response and tumour progression. Further research into the diagnostic and therapeutic implications of our findings is warranted.

\section{References}

1. Jemal A, Bray F, Center MM, Ferlay J, Ward E and Forman D: Global cancer statistics. CA Cancer J Clin 61: 69-90, 2011.

2. Manfredi S, Lepage C, Hatem C, Coatmeur O, Faivre J and Bouvier AM: Epidemiology and management of liver metastases from colorectal cancer. Ann Surg 244: 254-259, 2006.

3. Ramia JM, Lopez-Andujar R, Torras J, et al: Multicentre study of liver metastases from colorectal cancer in pathological livers. HPB (Oxford) 13: 320-323, 2011.

4. Martinez-Outschoorn UE, Lin Z, Trimmer C, et al: Cancer cells metabolically 'fertilize' the tumor microenvironment with hydrogen peroxide, driving the Warburg effect: implications for PET imaging of human tumors. Cell Cycle 10: 2504-2520, 2011.

5. Hamaya K, Hashimoto $\mathrm{H}$ and Maeda Y: Metastatic carcinoma in cirrhotic liver - statistical survey of autopsies in Japan. Acta Pathol Jpn 25: 153-159, 1975.

6. King PD and Perry MC: Hepatotoxicity of chemotherapy. Oncologist 6: 162-176, 2001.

7. Rubbia-Brandt L, Audard V, Sartoretti P, et al: Severe hepatic sinusoidal obstruction associated with oxaliplatin-based chemotherapy in patients with metastatic colorectal cancer. Ann Oncol 15: 460-466, 2004.

8. Kandutsch S, Klinger M, Hacker S, Wrba F, Gruenberger B and Gruenberger T: Patterns of hepatotoxicity after chemotherapy for colorectal cancer liver metastases. Eur J Surg Oncol 34: 1231-1236, 2008.

9. Cleary JM, Tanabe KT, Lauwers GY and Zhu AX: Hepatic toxicities associated with the use of preoperative systemic therapy in patients with metastatic colorectal adenocarcinoma to the liver. Oncologist 14: 1095-1105, 2009.

10. Ryan P, Nanji S, Pollett A, et al: Chemotherapy-induced liver injury in metastatic colorectal cancer: semiquantitative histologic analysis of 334 resected liver specimens shows that vascular injury but not steatohepatitis is associated with preoperative chemotherapy. Am J Surg Pathol 34: 784-791, 2010.

11. Tamandl D, Klinger M, Eipeldauer S, et al: Sinusoidal obstruction syndrome impairs long-term outcome of colorectal liver metastases treated with resection after neoadjuvant chemotherapy. Ann Surg Oncol 18: 421-430, 2011.

12. Rizzo G and Fiorucci S: PPARs and other nuclear receptors in inflammation. Curr Opin Pharmacol 6: 421-427, 2006.

13. Peters JM, Shah YM and Gonzalez FJ: The role of peroxisome proliferator-activated receptors in carcinogenesis and chemoprevention. Nat Rev Cancer 12: 181-195, 2012.

14. Dindo D, Demartines N and Clavien PA: Classification of surgical complications: a new proposal with evaluation in a cohort of 6336 patients and results of a survey. Ann Surg 240: 205-213, 2004.

15. Vauthey JN, Pawlik TM, Ribero D, et al: Chemotherapy regimen predicts steatohepatitis and an increase in 90-day mortality after surgery for hepatic colorectal metastases. J Clin Oncol 24: 2065-2072, 2006.

16. Mehta NN, Ravikumar R, Coldham CA, et al: Effect of preoperative chemotherapy on liver resection for colorectal liver metastases. Eur J Surg Oncol 34: 782-786, 2008.

17. Nakano H, Oussoultzoglou E, Rosso E, et al: Sinusoidal injury increases morbidity after major hepatectomy in patients with colorectal liver metastases receiving preoperative chemotherapy. Ann Surg 247: 118-124, 2008.

18. Di Paola R and Cuzzocrea S: Peroxisome proliferator-activated receptors ligands and ischemia-reperfusion injury. Naunyn Schmiedebergs Arch Pharmacol 375: 157-175, 2007 (In Chinese).

19. Braissant O, Foufelle F, Scotto C, Dauca M and Wahli W: Differential expression of peroxisome proliferator-activated receptors (PPARs): tissue distribution of PPAR-alpha, -beta, and -gamma in the adult rat. Endocrinology 137: 354-366, 1996.

20. Vidal-Puig AJ, Considine RV, Jimenez-Linan M, et al: Peroxisome proliferator-activated receptor gene expression in human tissues. Effects of obesity, weight loss, and regulation by insulin and glucocorticoids. J Clin Invest 99: 2416-2422, 1997.

21. Naishadham D, Lansdorp-Vogelaar I, Siegel R, Cokkinides V and Jemal A: State disparities in colorectal cancer mortality patterns in the United States. Cancer Epidemiol Biomarkers Prev 20: 1296-1302, 2011.

22. Kong L, Ren W, Li W, Zhao S, Mi H, Wang R, Zhang Y, Wu W, Nan Y and Yu J: Activation of peroxisome proliferator activated receptor alpha ameliorates ethanol induced steatohepatitis in mice. Lipids Health Dis 10: 246, 2011. 
23. Akiyama TE, Nicol CJ, Fievet C, et al: Peroxisome proliferator-activated receptor-alpha regulates lipid homeostasis, but is not associated with obesity: studies with congenic mouse lines. J Biol Chem 276: 39088-39093, 2001.

24. Stienstra R, Mandard S, Patsouris D, Maass C, Kersten S and Muller M: Peroxisome proliferator-activated receptor alpha protects against obesity-induced hepatic inflammation. Endocrinology 148: 2753-2763, 2007.

25. Okaya T and Lentsch AB: Peroxisome proliferator-activated receptor-alpha regulates postischemic liver injury. Am J Physiol Gastrointest Liver Physiol 286: G606-G612, 2004.

26. Pozzi A, Ibanez MR, Gatica AE, et al: Peroxisomal proliferatoractivated receptor-alpha-dependent inhibition of endothelial cell proliferation and tumorigenesis. J Biol Chem 282: 17685-17695, 2007.
27. Grau R, Diaz-Munoz MD, Cacheiro-Llaguno C, Fresno M and Iniguez MA: Role of peroxisome proliferator-activated receptor alpha in the control of cyclooxygenase 2 and vascular endothelial growth factor: involvement in tumor growth. PPAR Res 2008: $352437,2008$.

28. Kaipainen A, Kieran MW, Huang S, et al: PPARalpha deficiency in inflammatory cells suppresses tumor growth. PLoS One 2: e260, 2007 